\title{
The value of a CT scan compared to plain radiographs for the classification and treatment plan in tibial plateau fractures
}

\author{
Martijn A. J. te Stroet • Micha Holla • Jan Biert • \\ Albert van Kampen
}

Received: 18 October 2010 /Accepted: 23 December 2010/Published online: 11 March 2011

(C) The Author(s) 2011. This article is published with open access at Springerlink.com

\begin{abstract}
This study aimed to evaluate the intra- and interobserver agreement for both fracture classification according to Schatzker and treatment plan of tibial plateau fractures using plain radiographs alone and with computed tomography (CT) scans. The study was carried out prospectively to assess the impact of an advanced radiographic study on the agreement of treatment plan and fracture classification of tibial plateau fractures. Eight experienced observers (six surgeons and two radiologists) classified 15 tibial plateau fractures with plain radiographs and CT scans and set up a treatment plan. Agreement was measured using kappa coefficients. Using plain radiographs alone, the mean interobserver kappa coefficient for classification was 0.47 , which decreased to 0.46 after addition of CT scans. Using plain films alone for formulating a treatment plan, the mean interobserver kappa coefficient was 0.40 , which decreased to 0.30 after addition of $\mathrm{CT}$ scans. The mean intraobserver kappa coefficient for fracture classification using plain radiographs was 0.60 , which decreased to 0.57 with addition of CT scans. The mean intraobserver kappa coefficient for treatment plan based on plain radiographs alone was 0.53 , which decreased to 0.45 after addition of $\mathrm{CT}$ scans. In contrast with other recent
\end{abstract}

\footnotetext{
M. A. J. te Stroet $(\square) \cdot$ M. Holla $\cdot$ J. Biert $\cdot$ A. van Kampen

Radboud University Nijmegen Medical Centre,

Postbox 9101, 6501 HB, Nijmegen, The Netherlands

e-mail: martijn_testroet@hotmail.com

M. Holla

e-mail: M.Holla@orthop.umcn.nl

J. Biert

e-mail: J.Biert@CHIR.umcn.nl

A. van Kampen

e-mail: A.vanKampen@orthop.umcn.nl
}

publications, there is no increase in inter- and intraagreement of a CT scan compared to plain radiographs for the classification and treatment plan in tibial plateau fractures. Routine CT scanning of the knee for tibial plateau fractures is not supported by this study.

Keywords CT scan · Plain radiographs · Tibial plateau fracture $\cdot$ Schatzker classification $\cdot$ Treatment plan

\section{Introduction}

Fractures of the tibial plateau are among the most challenging fractures in orthopedics to treat. To prevent posttraumatic osteoarthritis and to restore optimal joint function, the congruency of the articular surface, the stability, and the correct load distribution needs to be restored [1].

To achieve this goal, an accurate preoperative planning is essential. In addition to the conventional plain radiographs, the CT scan is increasingly used. However, limited studies are available that report additional value of a CT scan [2-4]. Considering the additional load of radiation to the patient and costs, a CT scan should only be used when justified by the clinical benefits $[5,6]$.

With this intra- and interobserver study, the value of the CT scan in addition to plain radiographs for the classification of tibial plateau fractures according to Schatzker and the preoperative treatment plan was investigated.

\section{Materials and methods}

To cover all types of the Schatzker classification (1-6) at least twice, 15 patients with tibial plateau fractures were 
selected from our clinic by two experienced surgeons, who did not participate as an observer in this study.

Observers were not informed about the distribution of the types. Both plain radiographs and CT scans had to be available for this study. None of the selected cases had an open fracture. The radiographs of eight male and seven female patients with a mean age of 45 years (range, 12-78 years) were included.

The plain radiographs and $\mathrm{CT}$ scans of all cases were presented independently to six surgeons and two radiologists, all working in our level 1 trauma center for several years. CT scans were two-dimensional reconstructions performed by multislice thickness of at least $2 \mathrm{~mm}$. No three-dimensional reconstructions were shown. The observers were allowed to use all Agfa's IMPAX Web1000 ${ }^{\circledR}$ application tools.

After presentation of the plain radiographs, the observers were asked to classify the fractures according to Schatzker [7]. This classification is most commonly used as a guide for treatment of these fractures $[8,9]$. Each increasing numeric category indicates increasing severity: increased energy imparted to the bone and worse prognosis. Management of types 1-3 centers on evaluating and repairing articular cartilage, the fracture-dislocation mechanism of type 4 increases the likelihood of injury to the peroneal nerve or popliteal vessels, and in types 5 and 6 , the soft tissue status dictates the need for provisional stabilization with external fixation before definitive surgery.[10] A description of the classification with an illustration was presented to all observers during the test (Fig. 1). Next, the surgeons were asked to set up a treatment plan by selecting multiple options considering the surgical approach, the type of reduction, and stabilization (Table 1). Finally, they were asked if they would consider a CT scan necessary. After presentation of the CT scan, the classification was reconsidered, as well as the treatment plan.
Table 1 The multiple options for setting up the treatment plan for specific tibial plateau fractures

Possible treatment plan options (more options possible)

Functional non-weight bearing treatment

Long-leg cast non weight bearing immobilization

Lateral compression screw(s)

Medial compression screw(s)

Lateral plate

Medial plate

Percutaneous reduction of the lateral compartment with a punch

Percutaneous reduction of the medial compartment with a punch

Unilateral/Hoffmann fixator

Ilizarov fixator

One investigator supervised all observers to prevent them from changing answers of the classification and treatment plan based on plain radiographs, after studying the CT scan.

Three weeks later, the same cases were presented to the same observers in a different order.

A complete dataset was available for analysis. To define the intra- and interobserver agreement, the mean Cohen's kappa coefficient as described by Dunn was used [11] Kappa is a coefficient of agreement, which varies from +1 (perfect agreement) to 0 (agreement no better than chance) to -1 ( representing absolute disagreement).[9] These were calculated with the statistical programs SPPS ${ }^{\circledR}$ version 16.0 (SPSS, Inc., Chicago, IL, USA) and MedCalc ${ }^{\circledR}$ version 9.6.4.0 (MedCalc Software, Mariakerke, Belgium).

The results of the first reading were used to determine interobserver agreement. Cohen's kappa coefficients were interpreted according to Landis and Koch [12].
Fig. 1 The classification according to Schatzker divides the tibial plateau fractures into six types: lateral split fracture (1), lateral split fracture with depression (2), central depression fracture (3), medial condyle fracture (4), bicondylar fracture (5), and fracture with diaphysial discontinuity (6)
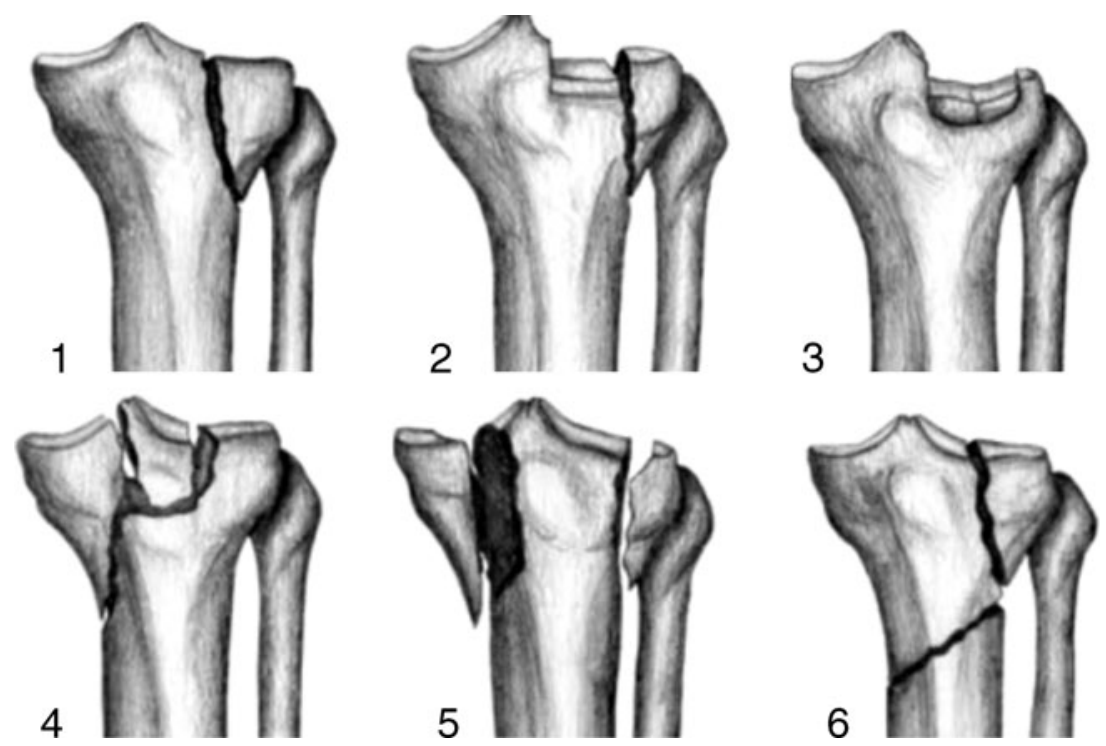


\section{Results}

The values for the intra- and interobserver agreement for the treatment subcategories are presented in Table 2.

\section{Intraobserver agreement}

The mean kappa coefficient for the Schatzker classification using plain films was 0.60 (range, 0.38 to 0.83 ) and with addition of the CT scan 0.57 (range, 0.43-0.74). Both values represent "moderate" intraobserver reliability (Fig. 2).

The mean kappa coefficient for the treatment plan based on plain radiographs was 0.53 (range, $0.40-0.65$ ); with a CT scan, this was 0.45 (range, 0.19-0.69). Both represent a "moderate" interobserver reliability (Fig. 3).

Interobserver agreement

The mean kappa coefficient for the Schatzker classification using plain radiographs was 0.47 (range, $0.13-0.74$ ), and with addition of the CT scans, it was 0.46 (range, 0.24-0.73). Both values represent a "moderate" interobserver reliability (Fig. 2).

The mean kappa coefficient for the treatment plan based on plain radiographs was 0.40 (range, $0.18-0.62$ ); with a CT scan, this was 0.30 (range, 0.11-0.56). Both represent a "fair" interobserver reliability (Fig. 3).

\section{Request for additional CT scan}

In $81 \%$ of all cases, the observers thought that an additional CT scan was appropriate.

\section{Discussion}

The results of this study did not show an increase in the inter- and intraobserver agreement for the fracture classifi-

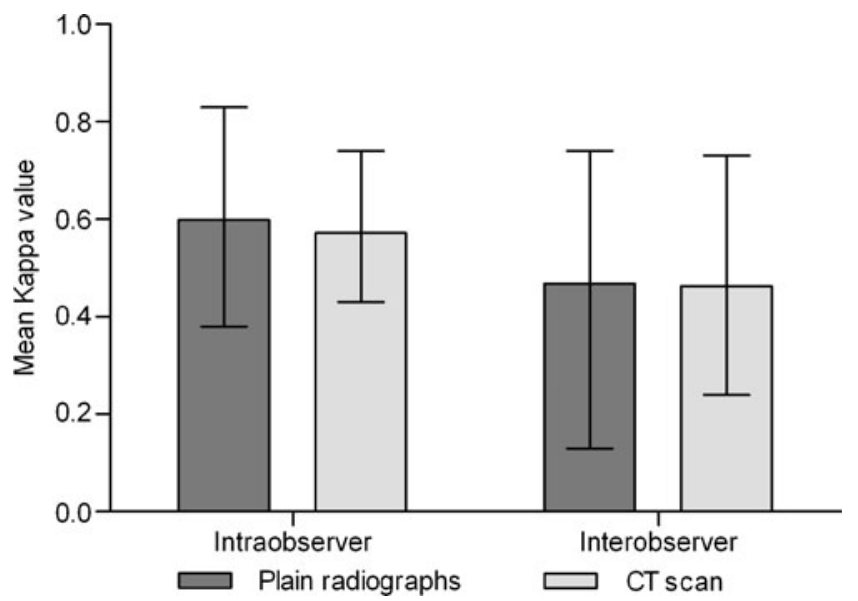

Fig. 2 Mean intra- and interobserver agreement over fracture classification according to Schatzker (with range)

cation of tibial plateau fractures with the addition of a CT scan.

One would hypothesize that $\mathrm{CT}$ scans would improve observer agreement because fracture lines and details are more clearly shown on these images [13]. Similar studies for the classification of proximal humeral or pelvic ring fractures, however, have also shown that CT scans did not improve interobserver agreement $[14,15]$.

Brunner et al. recently published a study with four observers and 45 retrospectively selected consecutive patients that demonstrates a better interobserver agreement [kappa value for plain radiographs $(0.42)$ vs. CT scan (0.76)] for classification of tibial plateau fractures with additional CT scans [2] However, patients who did not had CT scans as part of the initial fracture management were excluded from this study, an aspect that might cause bias as a result of unequal selection of types. For example, the differentiation between a Schatzker type 1 and 2 is more difficult with plain radiographs than between a Schatzker type 1 and 6 (Fig. 4). In contrast with Brunner, we selected
Table 2 Intra- and interobserver agreement over plain radiographs and CT scans for the classification, overall treatment and treatment subdivisions (with range)

\begin{tabular}{lll}
\hline & Plain radiographs & CT scan \\
\hline Intraobserver agreement & & \\
Classification according to Schatzker & $0.60(0.38-0.83)$ & $0.57(0.43-0.74)$ \\
Overall treatment & $0.53(0.40-0.65)$ & $0.45(0.19-0.69)$ \\
Anatomical approach & $0.60(0.37-0.82)$ & $0.53(0.43-0.74)$ \\
Type of fixation & $0.42(0.29-0.64)$ & $0.24(-0.13-0.48)$ \\
Method of reduction & $0.57(0.43-0.76)$ & $0.56(0.40-0.65)$ \\
Interobserver agreement & & $0.46(0.24-0.73)$ \\
Classification according to Schatzker & $0.47(0.13-0.74)$ & $0.30(0.11-0.56)$ \\
Overall treatment & $0.40(0.18-0.62)$ & $0.44(0.15-0.73)$ \\
Anatomical approach & $0.45(0.18-0.81)$ & $0.10(-0.20-0.36)$ \\
Type of fixation & $0.33(0.07-0.84)$ & $0.37(0.08-0.78)$ \\
Method of reduction & $0.42(0.05-0.77)$ & \\
\hline
\end{tabular}




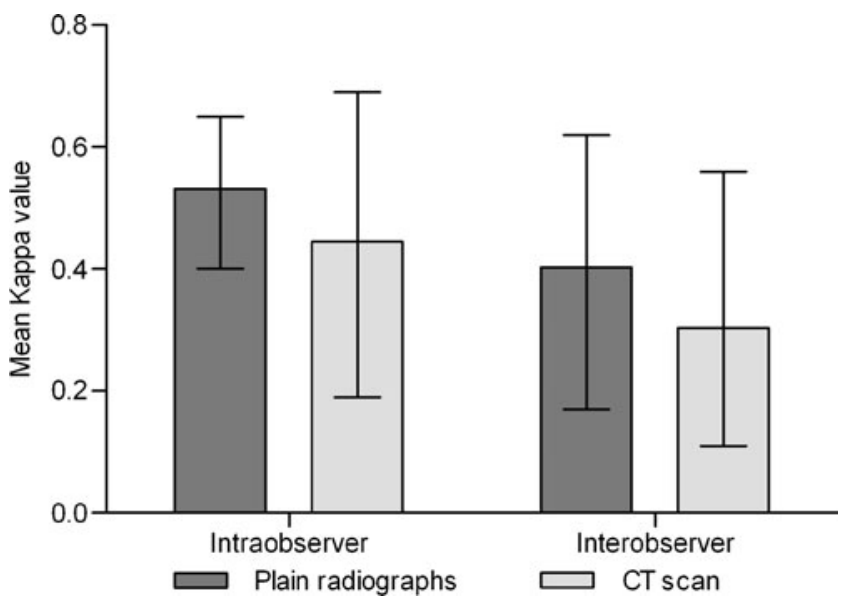

Fig. 3 Mean intra- and interobserver agreement over treatment plan for plain radiographs and CT scan (with range)

the whole range of types of fractures twice. Compared with that study, our set of selected cases was smaller (15 vs. 45$)$. However, we consulted more observers ( 8 vs. 4$)$, increasing the reliability and validity of our study.

In a previous similar study by Yacoubian, with three observers, the agreement for classification of tibial plateau fractures changed from 0.68 to 0.73 with the additional information of the CT scan [4].

Another study by Chan, with six observers, also did not show an increase in agreement for classification of tibial plateau fractures with the addition of a CT scan (kappa: plain, 0.62; CT scan, 0.61).[3]

A possible explanation, for the finding that an additional CT scan does not result in a better inter- and intraobserver agreement, is that the CT scan provides an overdose of information, which makes proper classification more difficult.

Furthermore, the classification according to Schatzker was developed in a time when CT scans were not readily available. Perhaps, a new classification based on CT findings would improve inter- and intraobserver agreement.
Furthermore, we studied the value of an additional CT scan in relation to the treatment plan. The results showed no increase in the inter- and intraobserver agreement for the treatment plan with the addition of a CT scan.

This is in contrast to an earlier publication by Chan that showed an increase in agreement for the treatment plan from 0.58 with plain radiographs to 0.71 with $\mathrm{CT}$ scans. Based on this finding, it was concluded that all patients with a tibial plateau fractures should have a CT scan.

A study by Yacoubian showed a slight improvement of the observer agreement from 0.72 with plain radiographs to 0.77 with CT scans. There are several possible reasons for our different findings.

The options of treatment plan of the study by Chan were limited to three treatment options. In our study, we used a multiple-option treatment plan with ten different modalities (Table 1). This may explain the lower inter- and intraobserver agreement. We believe that a precise description of multiple options for the treatment plan is more realistic.

The second reason can be the difference between the experience of the participating surgeons.

In other studies, observers with less training/residents were included as observer. In this study, all observers had at least 10 years of experience with interpretation of plain radiographs and clinical trauma surgery.

Making a CT scan can be potentially dangerous because of the use of ionizing radiation and therefore can cause cancer. In fact, in the USA alone, it is estimated that the use of CT scans accounts for around 6,000 additional cancers per year, with about half of those proving fatal [5].

Furthermore, cost-effectiveness is a factor that should be taken into consideration. Clear benefits must be proven to justify increased costs. Concerns arise when CT examinations are used without proven clinical rationale or when alternative modalities could be used with equal efficacy [6].

The clinical implication of the study is that surgeons should classify a tibial plateau fracture based on a series of plain radiographs and set up a treatment plan. Only if there
Fig. 4 The differentiation on AP plain radiographs, between Schatzker type 1 (a lateral split fracture) and type 2 (b lateral split fracture with depression) is more difficult than Schatzker type 1 and type 6 (c fracture with diaphysial discontinuity)
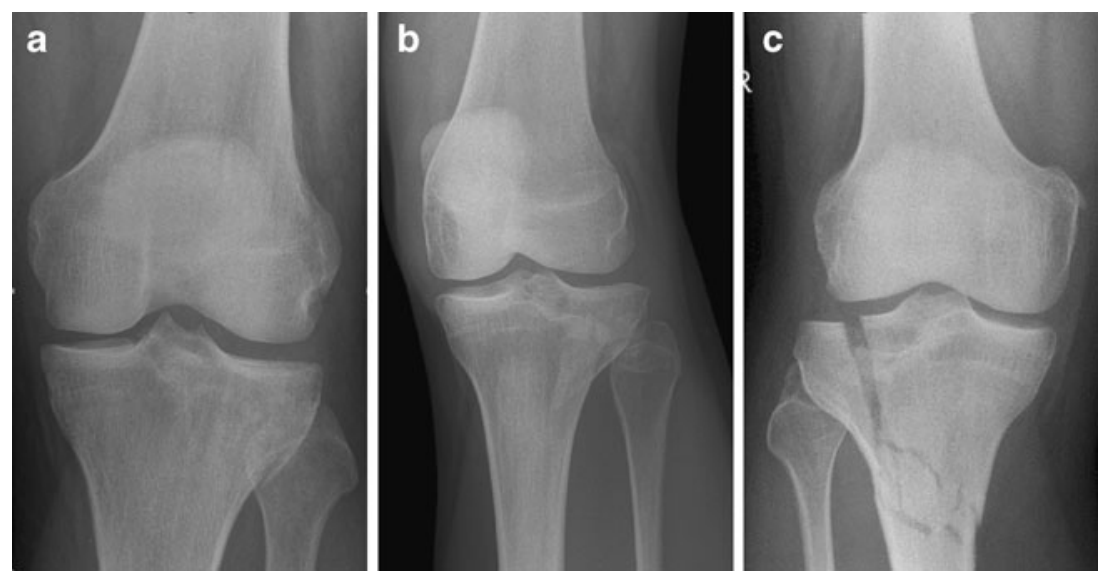
specific questions concerning the treatment plan remain that a CT scan should be considered. In our hospital, CT scans for tibial plateau fractures are not performed routinely anymore.

\section{Conclusions}

Concluding from this study, the use of a CT scan in addition to the plain radiographs in tibial plateau fractures did not result in a better intra- and interobserver agreement for the classification according to Schatzker and treatment plan. Considering additional costs and radiation, the routine use of a CT scan for tibial plateau fractures is questionable.

Open Access This article is distributed under the terms of the Creative Commons Attribution Noncommercial License which permits any noncommercial use, distribution, and reproduction in any medium, provided the original author(s) and source are credited.

\section{References}

1. Tscherne H, Lobenhoffer P (1993) Tibial plateau fractures. Management and expected results. Clin Orthop Relat Res 292:87-100

2. Brunner A, Horisberger M, Ulmar B et al (2010) Classification systems for tibial plateau fractures; does computed tomography scanning improve their reliability? Injury 41(2):173-178
3. Chan PSH, Klimkiewicz JJ, Luchetti WT et al (1997) Impact of CT scan on treatment plan and fracture classification of tibial plateau fractures. J Orthop Trauma 11(7):484-489

4. Yacoubian SV, Nevins RT, Sallis JG et al (2002) Impact of MRI on treatment plan and fracture classification of tibial plateau fractures. J Orthop Trauma 16(9):632-637

5. (2007) Radiation dose in computed tomography; why it's a concern and what you can do about it. Health Devices 36(2):41-63

6. Hall EJ, Brenner DJ (2008) Cancer risks from diagnostic radiology. Br J Radiol 81(965):362-378

7. Schatzker J, McBroom R, Bruce D (1979) The tibial plateau fracture. The Toronto experience 1968-1975. Clin Orthop Relat Res (138):94-104

8. Papagelopoulos PJ, Partsinevelos AA, Themistocleous GS et al (2006) Complications after tibia plateau fracture surgery. Injury 37 (6):475-484

9. Charalambous CP, Tryfonidis M, Alvi F et al (2007) Inter- and intraobserver variation of the Schatzker and AO/ATO classifications of tibial plateau fractures and proposal of a new classification system. Ann R Coll Surg Engl 89(4):400-404

10. Markhardt BK, Gross JM, Monu JU et al (2009) Schatzker classification of tibial plateau fractures: use of CT and MR imaging improves assessment. Radiographics 29(2):585-597

11. Dunn G (1989) Design and analysis of reliability studies. Oxford, New York

12. Landis JR, Koch GG (1977) The measurement of observer agreement for categorical data. Biometrics 33(1):159-174

13. Dirschl DR, Dawson PA (2004) Injury severity assessment in tibial plateau fractures. Clin Orthop Relat Res (423):85-92.

14. Bernstein J, Adler LM, Blank JE et al (1996) Evaluation of the Neer system of classification of proximal humeral fractures with computerized tomographic scans and plain radiographs. J Bone Joint Surg Am 78(9):1371-1375

15. Lansinger O, Bergman B, Körner L et al (1986) Tibial condylar fractures. A twenty-year follow-up. J Bone Joint Surg Am 68(1):13-19 